\title{
Parasitismo de Oreotrochilus leucopleurus (Trochilidae) en la Reserva Provincial de San Juan, Argentina
}

\author{
Castillo, G.N. ${ }^{1,2}$; Gonzalez, C.J.'; Acosta, J.C.,2 \\ ${ }^{1}$ Depto. Biología, Fac. Cs. Exactas Físicas y Naturales, Univ. Nac. San Juan, Argentina; ${ }^{2}$ Gab. Investig. Div.\& \\ Biol. Vertebr.; ${ }^{3}$ Centro Rehabilit. Fauna Silvestre, Av.Ignacio Roza 590, San Juan, Argentina. \\ nataliocastillo@gmail.com
}

\begin{abstract}
Resumen
Castillo, G.N.; Gonzalez, C.J.; Acosta, J.C.: Parasitismo de Oreotrochilus leucopleurus (Trochilidae) en la Reserva Provincial de San Juan, Argentina. Rev. Vet. 32: 2, 202-205, 2021. El conocimiento en ectoparásitos de aves silvestres es fragmentario, a pesar de que el parasitismo en este grupo de vertebrados es omnipresente. El ácaro rojo, Dermanyssus $s p$, es un parásito obligado de aves silvestres y domésticas. Ocasionalmente infesta mamíferos, incluidos perros, gatos, caballos, ganados y roedores. Este género representa gran importancia en medicina veterinaria para América Latina y cuya distribución se extiende en todo el mundo.En este sentido, el objetivo del trabajo fue determinar el ectoparasitismo en el picaflor andino Oreotrochilus leucopleurus en el centro oeste de Argentina. Se determinó la presencia de ninfas de Dermanyssus sp mediante muestras obtenidas de un nido de $O$. leucopleurus a 3500 metros de altitud. Nuestro estudio representa el primer registro de este parásito en $O$. leucopleurus, contribuyendo al conocimiento del parasitismo en aves silvestres.
\end{abstract}

Palabras clave: aves, ácaros, ectoparásitos, Trochilidae, San Juan, Argentina.

\begin{abstract}
Castillo, G.N.; Gonzalez, C.J.; Acosta, J.C.: Parasitism in Oreotrochilus leucopleurus (Trochilidae) from San Guillermo Provincial Reserve, San Juan, Argentina. Rev. Vet. 32: 2, 202-205, 2021. Knowledge about ectoparasites in wild birds is fragmentary, despite the fact that parasitism in this group of vertebrates is omnipresent. Dermanyssus sp. red mite, is an obligate parasite of wild and domestic birds. Occasionally it infests mammals, including dogs, cats, horses, cattle and rodents. This genus represents great importance in veterinary medicine for America Latin and whose distribution extends worldwide. Hence, the objective of this study was to determine the ectoparasitism in the andean humming bird Oreotrochilus leucopleurus in the center west of Argentina. We determined the nymphs' presence of Dermanyssus $s p$ through samples obtained from a nest of $O$. leucopleurus at 3500 meters of altitude. Our study represents the first record of this parasite in $O$. leucopleurus, contributing to knowledge in parasitism in wild birds.
\end{abstract}

Key words: birds, mites, ectoparasites, Trochilidae, San Juan, Argentina.

\section{INTRODUCCIÓN}

Entre los numerosos ectoparásitos aviares, el género Dermanyssus, particularmente Dermanyssus gallinae, especie citada en Argentina ${ }^{11}$, ocupa el lugar más importante en la producción avícola ${ }^{15,22}$. Este género representa gran importancia en medicina veterinaria principalmente en América Latina ${ }^{1}$.

Este parásito ha sido confirmado en 30 especies aviares ${ }^{19}$, entre salvajes y domésticas, además de encontrarse en 20 especies de mamíferos, incluido el hombre $15,21,22$. En seres humanos, la infestación provoca dermatitis y se ha reportado después de estar en contacto con aves ${ }^{11,21}$.

El género Dermanyssus actualmente incluye 23 especies, sin embargo, en la última revisión taxonómica, solo se consideraron 18 especies ${ }^{19}$. Dermanyssus $s p$ es un género de ácaros de hábitos hematófagos que parasita aves de corral, palomas, gorriones, canarios y jilgueros, entre otros ${ }^{6,11}$.

En ocasiones ataca a los mamíferos, incluyendo al hombre, siempre y cuando su hospedador usual no se encuentre disponible ${ }^{16,22}$. Por lo general, son de color grisáceo y se vuelven rojos cuando se llenan de sangre. La mayoría de las especies de Dermanyssus son nidícolas ${ }^{19}$. Las hembras adultas -proto y deutoninfas- poseen quelíceros distintivamente alargados y en forma de látigo ${ }^{6,19,22}$.

El ciclo básico de vida de los Dermanyssoidea incluye huevo, larva, dos estadios ninfales (proto y deutoninfa) y adulto. Las ninfas se diferencian de los adultos por la ausencia de aberturas genitales y tanto la proto como las deuto ninfas presentan placas y setas características ${ }^{11}$. 
Este ácaro está presente en el hospedador solo por la noche, cuando se alimenta. El resto del tiempo se localiza en grietas o en nidos de aves. Luego de alimentarse, las hembras colocan grupos de huevos en lugares ocultos en el nido. Las larvas no se alimentan hasta que se convierten en protoninfas, que mudan a adultos ${ }^{16}$.

El picaflor andino Oreotrochilus leucopleurus es una especie simpátrica con el picaflor puneño Oreotrochilus estella y el picaflor colorado Oreotrochilus adela. Esta especie presenta distribución prepuna, puna $\mathrm{y}$ estepas altoandinas. Se distribuye en todo el sector oeste de Argentina, desde la Provincia de Jujuy hasta el sur de Santa Cruz ${ }^{14}$

En la provincia de San Juan, O. leucopleurus ha sido mencionado para el Departamento de Calingasta ${ }^{8}$ y el Parque Nacional El Leoncito ${ }^{2}$. Según algunos, esta especie de ambientes Altoandino Puneños se caracteriza por encontrarse en zonas de vegas, siendo su estado de conservación de preocupación menor ${ }^{3}$.

El objetivo de este trabajo fue reportar el primer caso de ectoparasitismo en un ave silvestre de la Provincia de San Juan (Argentina), como así también el primer registro para este país de un ácaro del género Dermanyssus en $O$. leucopleurus, un picaflor andino común.

\section{MATERIAL Y MÉTODOS}

Área de estudio. Los muestreos se realizaron en febrero de 2017, en la Reserva Provincial San Guillermo, extremo norte del Departamento Iglesia, sector denominado Las Vegas del Ocucaro (-29.44586; -69.41617), Provincia de San Juan, Argentina (Figura 1). Este sector está incluido en la Cordillera Frontal, compuesto por materiales del Ordovícico, afloramientos de depósitos marinos del devónico ${ }^{12}$.

Biológicamente el área es de sumo interés, dominando elementos del Monte en los niveles inferiores a los $2.500 \mathrm{~m}$ de la Puna y Altoandinos, entre $3.000 \mathrm{~m} \mathrm{y}$ el límite de vegetación a 4.200-4.500 $\mathrm{m}^{12}$.

Los muestreos se realizaron en la región correspondiente a la Puna, donde dominan matorrales arbustivos
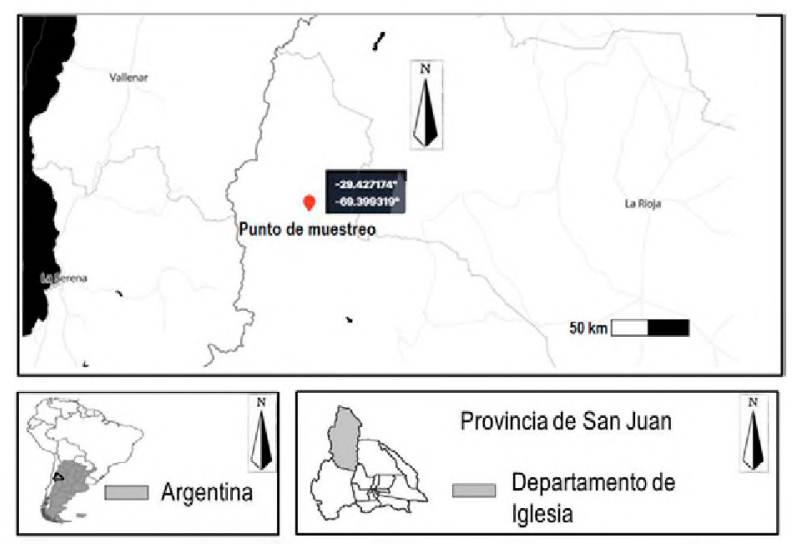

Figura 1. Ubicación geográfica del área de estudio: Reserva de Biosfera San Guillermo, San Juan, Argentina. bajos y medianos de Ephedra breana, Lycium tenuispinosum, cactáceas como Maihuenopsis glomerata, Lobivia formosa (Pfeiff.) y pastos de Stipa ichu y Aristida mendocina (Phil) aislados en estratos inferiores ${ }^{4}$.

Trabajo de campo y laboratorio. Se efectuó la revisión de un refugio humano abandonado (Figura 2), localizado en las proximidades de una vega a $3500 \mathrm{~m}$ de altura. Se registró la presencia del nido de un picaflor andino, Oreotrochilus leucopleurus (Trochilidae) (Figura 3). Se procedió a analizar y revisar cuidadosamente el nido en busca de ectoparásitos.

Los ácaros recolectados fueron colocados en un recipiente hermético y se trasladaron al laboratorio del Departamento de Biología (Facultad de Ciencias Exactas, Físicas y Naturales, Universidad Nacional de San Juan), donde se procedió a la identificación de los ácaros.

Se determinaron estadios ninfales del género $\mathrm{Der}$ manyssus sp (Figuras 4 y 5). Para la determinación se consultaron las principales claves propuestas $7,9,13,18,19$ . Además se consultaron las propuestas de los principales caracteres de identificación del género mencionados por ${ }^{6,10,17,22,24}$.

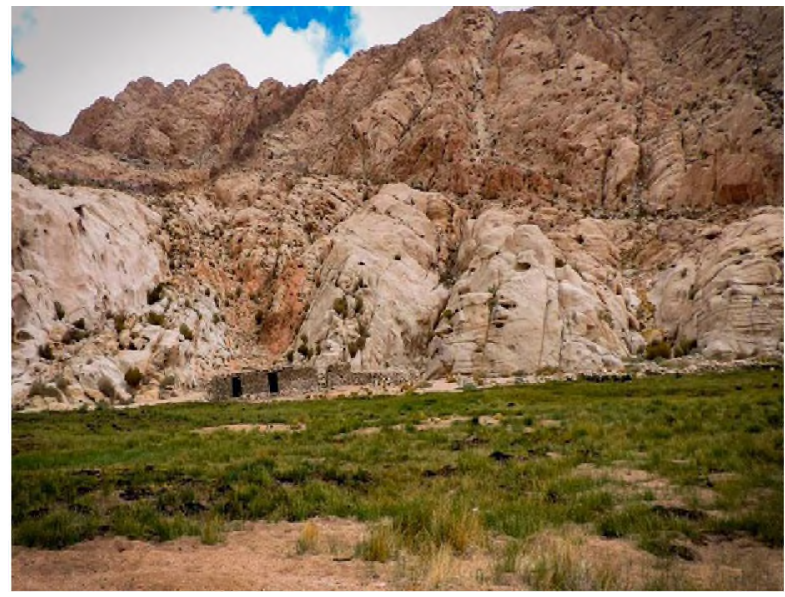

Figura 2. Área de estudio: reserva de biosfera San Guillermo, San Juan, República Argentina.

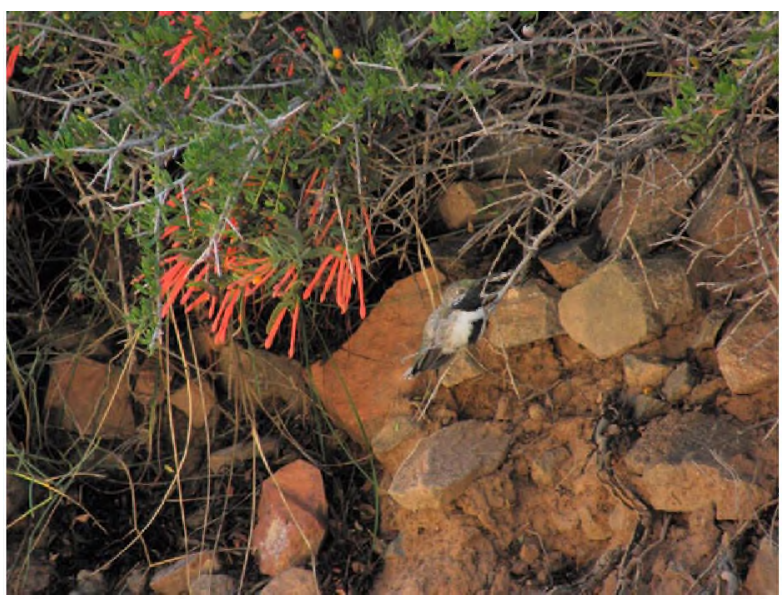

Figura 3. Ejemplar de Oreotrochilus leucopleurus, picaflor andino (fotografía del Dr. Gustavo Fava). 
Los ejemplares de Dermanyssus sp se encuentran depositados en la colección parasitológica del Gabinete de Investigación, Diversidad y Biología de Vertebrados del Árido, Departamento de Biología de la Facultad de Ciencias Exactas, Físicas y Naturales, Universidad Nacional de San Juan.

\section{RESULTADOS Y DISCUSIÓN}

Se contabilizaron aproximadamente una cantidad de 200 ácaros, pertenecientes a estadios ninfales de Dermanyssus sp (Figuras 4 y 5). Los estudios sobre parasitismos en aves silvestres son escasos.

Hasta el momento el género Dermanyssus sp no había sido mencionado en ningún ave silvestre cordillerana en Argentina. Ello probablemente fue así porque no había sido estudiado su parasitofauna. A pesar de que el parasitismo es omnipresente en aves silvestres, su conocimiento actual es fragmentario ${ }^{23}$.

Uno de los principales problemas que se presenta a la hora de estudiar la parasitofauna es que las muestras de aves silvestres disponibles para el estudio suelen estar sesgadas por el método de recolección ${ }^{23}$. Debido a los actuales problemas sistemáticos y taxonómicos en el que se encuentra la especie, los autores presentan una revisión histórica del género Dermanyssus s $p^{19}$ donde actualmente se incluyen 23 especies.

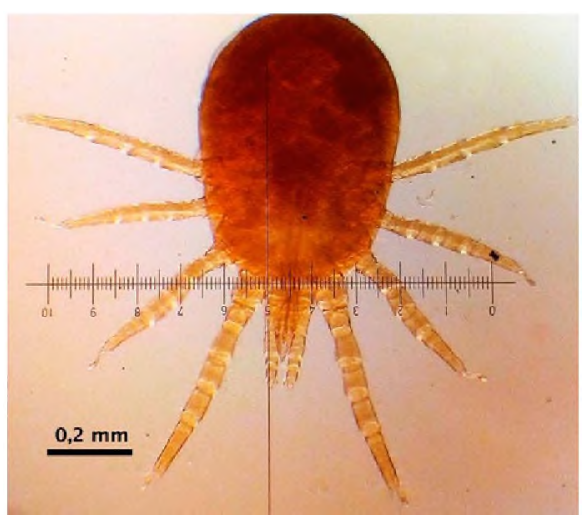

Figura 4. Estadio larval de Dermanyssus sp en el picaflor Oreotrochilus leucopleurus.

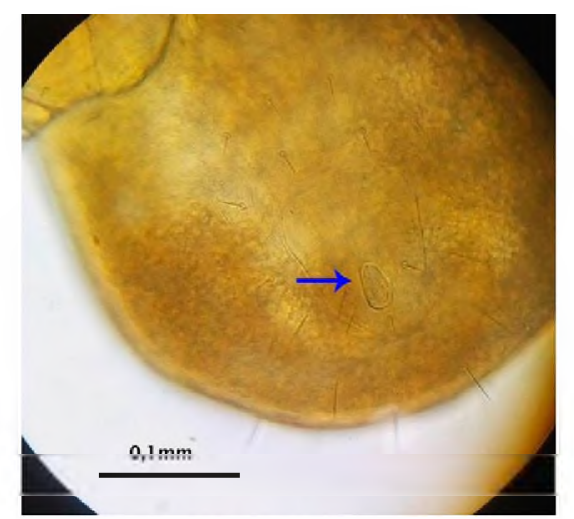

Figura 5. Estadios larvales de Dermanyssus sp en el picaflor Oreotrochilus leucopleurus. Vista ventral indicando la placa anal. La flecha señala el poro anal en el sector inferior de la placa anal.
En Argentina solo ha sido mencionado D. gallinae en tres especies de aves: Columba livia, Gallus gallus y Serinus canaria ${ }^{11}$. Dermanyssus gallinae es la especie más frecuentemente recolectada $\mathrm{y}$ aparentemente se encuentra en todo el mundo ${ }^{18}$

En el último listado propuesto por especialistas del tema ${ }^{19}$ se mencionan aproximadamente 30 especies de aves, distribuidas en 12 familias y 8 órdenes, parasitadas por $D$. gallinae. Entre las distintas especies del género, Dermanyssus trochilinis ha sido mencionado como parásito en aves de la familia Trochilidae ${ }^{18}$

Dentro de los ácaros ectoparásitos más dañinos para las aves de vida libre, se encuentran Dermanyssus $s p$ y Ornithonyssus sp. Ambos son extremadamente hematófagos y posibles transmisores de enfermedades, pudiendo ocasionar gran daño al permanecer escondidos en los nidos, multiplicándose rápidamente en gran número e ingiriendo crecidos volúmenes de sangre del hospedero $^{20}$.

Ambos géneros pueden ser posiblemente confundidos, salvo ciertas diferencias en el ciclo y características morfológicas. La familia Dermanyssidae se caracteriza por presentar el idiosoma ampliamente redondeado en sentido posterior y el segundo artículo queliceral de la hembra alargado, muy superior al segmento basal en longitud. En Dermanyssus sp el ano se encuentra en el sector posterior a la placa anal, mientras que en Ornithonyssus sp el ano se encuentra en el sector anterior de dicha placa anal ${ }^{24}$.

El estudio detallado del nido del picaflor Oreotrochilus leucopleurus, determinó que el $90 \%$ del nido estaba construido con pelos de guanacos (Lama guanicoe) y vicuñas (Vicugna vicugna). La zona de recolección se caracteriza por la presencia de ambas especies de camélidos. El resto de la nidificación lo componen ramas, hojas y otro tipo de vegetación no identificada.

Nuestros datos coinciden con el reporte de nidificación del picaflor andino del Parque Nacional El Leoncito, Departamento Calingasta, Argentina ${ }^{2}$. Para nuestro caso de estudio, el nido se encontraba en el interior de un refugio humano abandonado, compuesto de rocas y vigas de maderas. Probablemente la morada fuera de puesteros que venían con ganado para realizar prácticas de pasturas.

El nido se encontraba ubicado en un lugar oculto, protegido y resguardado del frío. Si bien tal "alcoba" no se encontraba colgando del techo, realmente se hallaba sobre una de las vigas que formaba parte de la estructura del techado. El picaflor nidifica en grietas, cuevas y construcciones humanas, lugares ventajosas para la incubación de huevos y la regulación térmica de pichones ${ }^{5}$.

En conclusión, se realizó un aporte en el conocimiento parasitológico de las aves silvestres cordilleranas para la Provincia de San Juan, Argentina. Actualmente no existe ninguna mención sobre los ectoparásitos que contribuyan a la información sobre el estado sanitario en el que se encuentran tales aves, con referencia al parasitismo. La mayoría de los estudios 
en aves se encuentran sesgados a la distribución y aspectos ecológicos. El presente estudio realiza una contribución sobre este tema.

Otra especie del género (Dermanyssus gallinae), ha comprobado ser un problema importante en las operaciones avícolas. Las infestaciones de los ácaros pueden ser debilitantes, provocando irritación y lesiones importantes, comúnmente observadas en las patas de aves, pérdida de vigor, retraso del crecimiento, pérdida de plumas, reducción de producción de huevos, anemia y -a veces- muerte, especialmente de aves jóvenes ${ }^{22}$.

Agradecimientos: a la Subsecretaría de Medio Ambiente de la Provincia de San Juan (Argentina) por los permisos otorgados. Al equipo técnico y guardaparques de la Reserva de Biosfera San Guillermo de San Juan. Al Dr. Gustavo Fava por las fotografías de Oreotrochilus leucopleurus.

\section{REFERENCIAS}

1. Barriga OO. 2002. Las enfermedades parasitarias de animales domésticos en América Latina, Editorial Germinal, Chile.

2. Bodrati A, Merida E, Montenegro L. 2003. Nidificación del picaflor andino común (Oreotrochilus leucopleurus) en el Parque Nacional El Leoncito, Provincia de San Juan, Argentina. Nuestras Aves 45: 26-28.

3. Birdlife International. 2016. Oreotrochilus leucopleurus The IUCN Red List of Threatened Species. ISSN 23078235 (on line).

4. Cabrera AL, Willink A. 1973. Biogeografía de América latina. The General Secretariat of the Organization of American States, Washington USA.

5. Carpenter LF. 1976. Ecology and evolution of an andean hummingbird (Oreotrochilusestella). Univ Calif Publ Zool 106: 1-74.

6. Cordero CM, Rojo FA. 2001. Parasitología veterinaria. McGraw-Hill Interamericana, Madrid.

7. Dipalma A, Giangaspero A, Cafiero MA, Germinara G. 2012. A gallery of the key characters to ease identification of Dermanyssus gallinae (Acari: Gamasida: Dermanyssidae) and allow differentiation from Ornithonyssus sylviarum (Acari: Gamasida: Macronyssidae). Parasites \& vectors 5: 104 .

8. Fava GA, Acosta JC, Laspiur JA, Moreno MV. 2012. Avifauna del Valle de Calingasta y sus alrededores, San Juan, Argentina. Acta Zool Lilloana 56: 66-81.
9. Knee W, Proctor H. 2006. Keys to the families and genera of blood and tissue feeding mites associated with $\mathrm{Al}$ bertan birds. Can J Arthropod Identif 2: 1-18.

10. González A, Lareschi M. 2005. Acari (excepto Ixodida). En: Salomón OD, Ed. Artrópodos de Interés Médico en Argentina, $1^{\text {a }}$ ed., Buenos Aires, p. 7-14.

11. Lareschi M, Mauri R. 1998. Dermanyssoidea. En: Morrone JJ, Coscarón $\mathrm{S}$, ed. Biodiversidad de Artrópodos Argentinos, La Plata, Ediciones Sur, p. 581-590.

12. Martínez CE. 2007. Marco contextual. En: Diversidad biológica y cultural de los Altos Andes Centrales de Argentina, Univ. Nacional de San Juan, p.115-152.

13. Moss WW. 1978. The mite genus Dermanyssus: a survey with description of Dermanyssus trochilinis and a revised key to the species. JMed Entomol 14: 627-640.

14. Narosky S, Yzurieta D, Matarasso H. 2010. Aves de Argentina y Uruguay: guia de identificación, Editorial Oro, Buenos Aires, Argentina.

15. Pavlovic I, García T. 2014. Dermanyssus gallinae en la producción avícola. Albéitar 177: 26-27

16. Rosa A, Ribicich M. 2012. Parasitología y enfermedades parasitarias en veterinaria. Hemisferio Sur, Buenos Aires, Argentina, $330 \mathrm{p}$

17. Romero HQ. 1990. Parasitología y enfermedades parasitarias, Editorial Limusa, México, 854 pag.

19. Roy L, Chauve CM. 2007. Historical review of the genus Dermanyssus, Duges 1834 (Acari: Mesostigmata: Dermanyssidae). Parasite 14: 87-100.

18. Roy L, Chauve CM. 2009. The genus Dermanyssus (Mesostigmata: dermanyssidae): history and species characterization. En: Sabelis MW, Bruin J. eds. Trends in Acarology, Springer, 49-55

20. Soto CJ, Eliéser LC, Acosta GI, Aguilera X, Correa M. 2007.Repercusión de la presencia de ectoparásitos hematófagos en el cuad ro hematológicos de pichones de cotorra silvestre. Redvet 8: 1-9.

21. Téllez ML, Sordo $C$, Ruiz A, Tucto $S$, Manrique A. 2008. Dermatosis por ácaros de palomas. Primer reporte de la presencia de Ornithonyssus sylviarum en el Perú. Folia Dermatol 19: 63-68.

22. Williams RE. 2010. Veterinary Entomology, Livestock and Companion Animals. Editorial Taylor and Francis Group, Indiana, U.S.

23. Wobeser GA. 2008. Parasitism: costs and effects. En: Atkinson TC, Bruce NJ, eds. Parasitic diseases of wild birds. Ed. John Wiley \& Sons, p. 3-9.

24. Zajac MA, Conboy GA. 2006. Veterinary Clinical Parasitology. Blackwell Publishing, Australia. 